\title{
BMJ Global Health Using research to prepare for outbreaks of severe acute respiratory infection
}

\author{
For the SPRINT-SARI investigators
}

\begin{abstract}
To cite: For the SPRINT-SARI investigators. Using research to prepare for outbreaks of severe acute respiratory infection. BMJ Glob Health 2019;4:e001061. doi:10.1136/ bmjgh-2018-001061
\end{abstract}

Handling editor Seye Abimbola

Received 17 July 2018

Revised 17 September 2018

Accepted 6 0ctober 2018

Check for updates

\section{(C) Author(s) (or their} employer(s)) 2019. Re-use permitted under CC BY-NC. No commercial re-use. See rights and permissions. Published by BMJ.

Correspondence to

Srinivas Murthy;

srinivas.murthy@cw.bc.ca

\section{ABSTRACT}

Severe acute respiratory infections (SARI) remain one of the leading causes of mortality around the world in all age groups. There is large global variation in epidemiology, clinical management and outcomes, including mortality. We performed a short period observational data collection in critical care units distributed globally during regional peak SARI seasons from 1 January 2016 until 31 August 2017, using standardised data collection tools. Data were collected for 1 week on all admitted patients who met the inclusion criteria for SARI, with follow-up to hospital discharge. Proportions of patients across regions were compared for microbiology, management strategies and outcomes. Regions were divided geographically and economically according to World Bank definitions. Data were collected for 682 patients from 95 hospitals and 23 countries. The overall mortality was $9.5 \%$. Of the patients, $21.7 \%$ were children, with case fatality proportions of $1 \%$ for those less than 5 years. The highest mortality was in those above 60 years, at $18.6 \%$. Case fatality varied by region: East Asia and Pacific 10.2\% (21 of 206), Sub-Saharan Africa 4.3\% (8 of 188), South Asia 0\% (0 of 35), North America 13.6\% (25 of 184), and Europe and Central Asia $14.3 \%$ (9 of 63 ). Mortality in low-income and low-middle-income countries combined was $4 \%$ as compared with $14 \%$ in high-income countries. Organ dysfunction scores calculated on presentation in 560 patients where full data were available revealed Sequential Organ Failure Assessment (SOFA) scores on presentation were significantly associated with mortality and hospital length of stay. Patients in East Asia and Pacific (48\%) and North America (24\%) had the highest SOFA scores of $>12$. Multivariable analysis demonstrated that initial SOFA score and age were independent predictors of hospital survival. There was variability across regions and income groupings for the critical care management and outcomes of SARI. Intensive care unit-specific factors, geography and management features were less reliable than baseline severity for predicting ultimate outcome. These findings may help in planning future outbreak severity assessments, but more globally representative data are required.

\section{INTRODUCTION}

Lower respiratory infections are the leading infectious cause of death worldwide. ${ }^{1}$ Severe acute respiratory infection (SARI) is an acute respiratory infection with fever, cough and hospitalisation and is used for surveillance

\section{Summary box}

Severe acute respiratory infections (SARI) are one of the leading global causes of mortality and the most likely syndrome associated with future pandemics.

- Research preparedness for future pandemics includes demonstrating global collaboration, as well as understanding the baseline clinical features of the disease.

- Baseline severity of illness scores are highly predictive of outcomes.

- Global collaboration across regions with standardised data collection is feasible, and rapid dissemination of data during a future pandemic through this platform holds great promise.

- Future research should refine data elements collected and expand sampling at disparate regions to improve research preparedness for future pandemics.

in acute care settings globally. Organ-specific support is often required, with high attributable mortality. ${ }^{2}$ A better understanding of global variability in the epidemiology and care provided for critically ill patients with SARI is lacking.

The SPRINT-SARI (Short Period prevalence Study of Severe Acute Respiratory Infection) programme leverages the function of existing networks that have been established to provide a rapid research response in the event of a future respiratory outbreak of public health importance.

As part of the SPRINT-SARI and International Severe Acute Respiratory and Emerging Infection Consortium networks of clinical research preparedness for future SARI outbreaks, we are collecting period prevalence data for SARI across disparate research networks and hospitals around the world. The primary aim in establishing this network is to create a global collection of hospitals, from disparate regions and hospital types, primed to collect observational data on patients with SARI in a future outbreak. ${ }^{3}$ This involves annual data collection rehearsals to both better understand epidemiology over time and to maintain institutional familiarity 
with the process of clinical research data collection. ${ }^{4}$ The importance of preapproved protocols for research during emergencies has been well demonstrated. ${ }^{56}$ This is the first time that the global readiness to conduct such outbreak-related research has been repeatedly tested using a common data set among many global regions.

In addition to research preparedness for future outbreaks, documenting variation in epidemiology, management and outcomes of patients with SARI across regions is the first step towards standardising care, improving outcomes, and ensuring that interventional studies are performed on the background of optimal, standardised clinical care. We present combined results from the first two seasons of data collection for this programme during 2016-2017, where the primary circulating respiratory viruses were influenza A (H3N2) and $\mathrm{A}(\mathrm{H} 1 \mathrm{~N} 1) \mathrm{pdm} 0{ }^{78}$

\section{ROLL-OUT OF DATA COLLECTION INFRASTRUCTURE Design}

SPRINT-SARI is an annual period prevalence study enrolling newly admitted patients with SARI to participating intensive care units or hospital wards where intensive care units were not accessible. All patients admitted over a 1-week period during the peak respiratory infection season in northern and southern hemispheres were enrolled. Hospitals and intensive care units were sampled through convenience, due to their participation in prior studies or involvement with ongoing research networks. This study was registered at ClinicalTrials.gov (NCT02498587).

\section{Patients}

Eligible patients were those admitted to the relevant hospital unit of interest if they met the following inclusion criteria: suspected or proven acute respiratory infection with onset within the past 14 days, with one or more of the following: fever, cough, dyspnoea or tachypnoea. There were no exclusion criteria. Consent was obtained where deemed appropriate per local ethics boards, and waived at other institutions due to the routine and anonymised nature of the data collected. ${ }^{9}$

\section{Data collected}

Clinical data were collected prospectively at participating centres, and consisted of presenting signs and symptoms, severity of illness, comorbidities, interventions received during hospitalisation and clinical outcomes at time of discharge. Pathogen testing was according to routine diagnostic practices at each participating centre. Organ dysfunction for adults was determined through calculating the Sequential Organ Failure Assessment (SOFA) scores. ${ }^{10}$ For children, an adapted organ dysfunction score was used to capture rates of organ dysfunction. ${ }^{11}$ Scores were calculated from worst reported component data obtained on the first day of admission. Outcomes were not censored for the duration of stay. Data collection was facilitated through open-access clinical protocols, case report forms and web-based data entry of fully anonymised data through secure servers.

\section{Analysis}

The data were first checked for completeness and missing values. Data explorations were performed by running frequency checks and descriptive statistics. Data on primary study variables (outcomes, exposure metrics and derived covariates) were summarised using descriptive statistics, including measures of central tendency and dispersion for continuous variables (mean and SD, median and IQR) and frequencies with proportions for categorical variables. Univariate and multivariate logistic regressions were used to assess the association between mortality and main a priori proposed risk factors or adjusting for potential confounders. All statistical significance was assessed at the $5 \%$ level of significance (two-sided $\mathrm{p}<0.05$ ). Analyses were performed using STATA V.14 statistical software. Regions were defined by income and geography according to World Bank country and lending groups criteria. ${ }^{12}$

\section{CRITICAL ILLNESS DUE TO SEVERE ACUTE RESPIRATORY INFECTIONS}

Data were collected from 1 January 2016 until 31 August 2017, from 682 patients admitted to 95 participating hospitals in 20 countries (see table 1 and figure 1 ). There was a bimodal distribution of age, with the majority of the sample children less than 10 years of age $(30.0 \%)$ and those above $60(39.9 \%)$ years $(27.7 \%)$ (see figure 2$)$.

The overall mortality was $9.5 \%$. The lowest case fatality proportion was in children under 5 years $(1 \%)$. The highest case fatality proportions were in adults over 60 years: $18.4 \%$ mortality in patients aged $60-79$ years and $18.9 \%$ in those $>80$ years. Mortality in low-income/ low-middle-income countries was 4\% (12 of 302) and was $14 \%$ (53 of 380 ) in high-income countries. Mortality varied between geographical regions, with East Asia and Pacific at $10.2 \%$, Sub-Saharan Africa 4.3\%, South Asia at $0 \%$, North America $13.6 \%$, and Europe and Central Asia $14.3 \%$. The availability of higher level care in intensive care-type settings was variable across regions, with access to ventilators absent in eight sites in low-income/ low-middle-income countries. This possibly explains the unexpected variability in mortality, where less-sick patients were enrolled in Sub-Saharan Africa, given the lack of formal intensive care units in the region.

\section{CRITICAL CARE INTERVENTIONS}

When examining interventions used, patients in East Asia $(50.7 \%)$, North America $(49.3 \%)$ and Europe (35.6\%) had higher frequencies of invasive mechanical ventilation compared with patients in South Asia (4.4\%) and Sub-Saharan Africa (14.3\%). Dialysis was received in 0\% in Sub-Saharan Africa and Latin America and the Caribbean, $8.3 \%$ in Europe and Central Asia, 9.3\% in North America, and $6.7 \%$ in East Asia and Pacific, although with 
Table 1 Demographics of included patients $(n=682)$

\begin{tabular}{|c|c|}
\hline \multicolumn{2}{|l|}{ Characteristics } \\
\hline Age (median) & 49 (IQR 3-70) \\
\hline Female (\%) & 44.5 \\
\hline $\begin{array}{l}\text { Duration of symptoms before hospitalisation } \\
\text { (days) }(n=682)\end{array}$ & $3($ IQR 1-5) \\
\hline \multicolumn{2}{|l|}{ Number of listed comorbidities (\%) } \\
\hline 0 & $473(69.4)$ \\
\hline 1 & $76(11.1)$ \\
\hline 2 & $72(10.6)$ \\
\hline 3 & $34(5.0)$ \\
\hline $4+$ & $27(4.0)$ \\
\hline \multicolumn{2}{|l|}{ Income group (\%) } \\
\hline Low income & $181(26.5)$ \\
\hline Lower middle income & $121(17.7)$ \\
\hline Upper middle income & $3(0.2)$ \\
\hline High income & $377(55.3)$ \\
\hline \multicolumn{2}{|l|}{ Geographical group (\%) } \\
\hline Sub-Saharan Africa & $188(27.6)$ \\
\hline East Asia and Pacific & $206(30.2)$ \\
\hline South Asia & $35(5.1)$ \\
\hline North America & $184(27.0)$ \\
\hline Europe and Central Asia & $63(9.2)$ \\
\hline Middle East and North Africa & $3(0.4)$ \\
\hline Latin America and the Caribbean & $3(0.4)$ \\
\hline \multicolumn{2}{|l|}{ Age group $(\%)$} \\
\hline $0-10$ & $205(30.0)$ \\
\hline $11-20$ & $24(3.5)$ \\
\hline $21-30$ & $30(4.4)$ \\
\hline $31-40$ & $32(4.6)$ \\
\hline $41-50$ & 52 (7.6) \\
\hline $51-60$ & $63(9.2)$ \\
\hline $61-70$ & $103(15.1)$ \\
\hline $71-80$ & $80(11.7)$ \\
\hline$>80$ & $83(12.1)$ \\
\hline
\end{tabular}

small sample sizes and variable availabilities of interventions.

\section{SEVERE ACUTE RESPIRATORY INFECTION PATHOGENS}

The proportion of patients without a documented infectious aetiology was $66.9 \%$ (456 of 682 ), of whom $7.9 \%$ (36 of 456) died, as defined as no pathogen was deemed causative by the clinical team. Complete diagnostic testing was not available in many of the regions, with no viral testing used in low-income or low-middle-income regions; most of the patients with a documented infection were from high-income settings. The most commonly diagnosed pathogen was influenza virus (11.6\%, 77

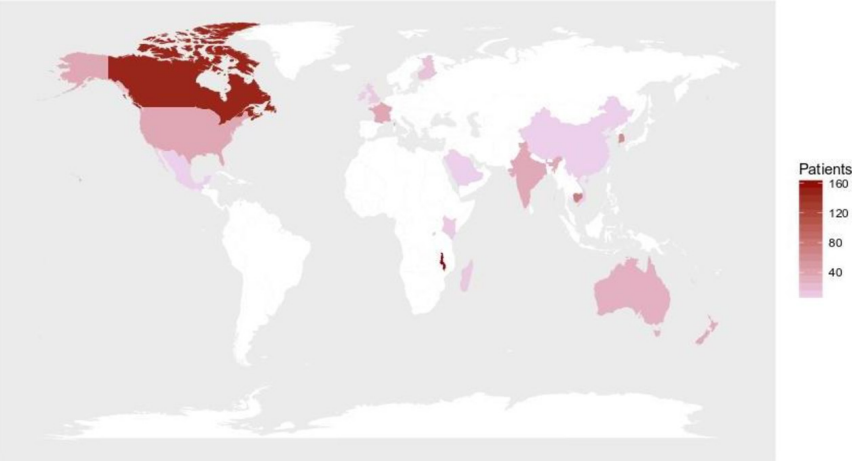

Figure 1 Countries where there are SPRINT-SARI data collection sites, by numbers of patients recruited. SPRINTSARI, Short Period prevalence Study of Severe Acute Respiratory Infection.

of 663). Respiratory syncytial virus was identified in 19 patients $(2.7 \%)$, with $<1 \%$ of patients with coronavirus $(0.92 \%, 6$ of 655$)$ and none with Middle East respiratory syndrome-coronavirus in this cohort. Bacterial infections were identified in 25 of 682 patients $(3.6 \%)$, with Streptococcus pneumoniae being the most frequently isolated bacterial pathogen in respiratory sampling $(n=8)$.

Pathogen-specific disease burden has been examined by a number of other groups. ${ }^{13}$ However, our finding that the majority of critically ill patients with SARI have no diagnosed microbial aetiology reinforces the need for syndrome-based, in addition to pathogen-based, surveillance for critically ill patients. This is especially true given the lack of relevant diagnostic testing in much of the world, which impacted the proportion of patients without identified pathogens. ${ }^{15}$ The availability of global data and collaboration is essential, given the likely international nature of future outbreaks and requirements for pre-established coordination efforts. ${ }^{16}$

The lack of a dedicated sampling protocol clearly impacts on interpretation of those findings. Future work should focus on optimising hospital sampling strategies to better understand global SARI disease; the lack of SARI pathogen-specific diagnostics on recently released Essential Diagnostic Lists exemplifies the lack of clarity on best testing strategies. ${ }^{17}$ Regional SARI surveillance data have been published from a variety of countries $^{18}{ }^{19}$; while we have yet to completely avoid site selection bias through

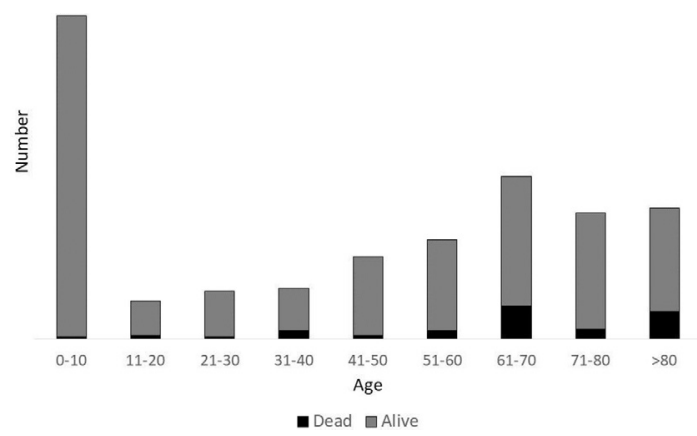

Figure 2 Age and case fatality proportion distribution of included patients. 


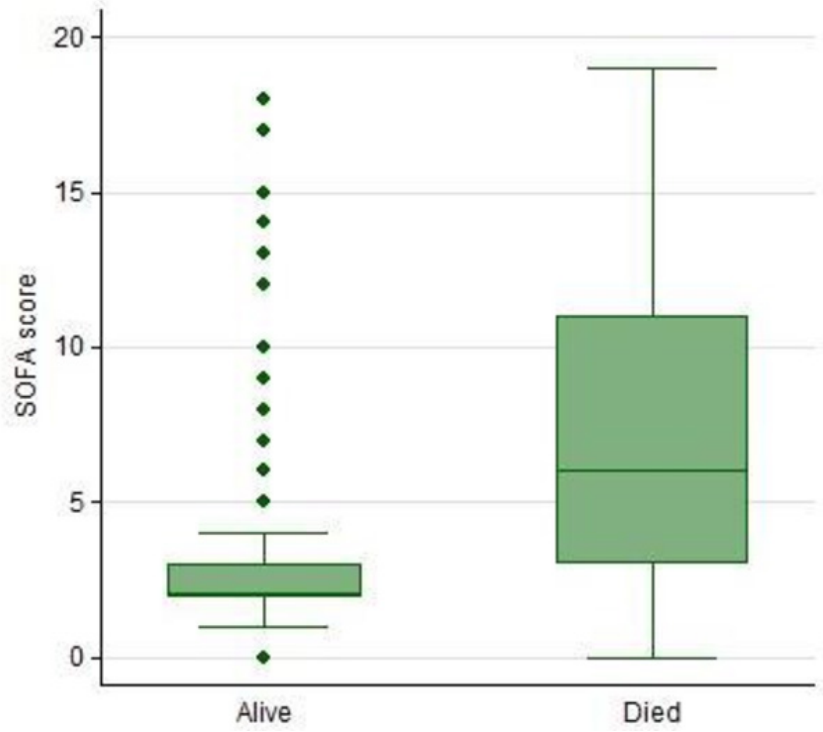

Figure 3 Hospital mortality and Sequential Organ Failure Assessment (SOFA) scores $(n=560)$.

the availability of reporting hospitals, the data collection tools used help reduce the risk of bias from individual patient selection or pathogen-specific attribution.

\section{SEVERITY OF ILLNESS DUE TO SEVERE ACUTE RESPIRATORY INFECTIONS}

Increasing SOFA score on presentation was associated with increasing mortality $(27 \%$ increase in mortality for every additional SOFA score point; figure 3 ). The highest organ dysfunction scores were in patients in the East Asia and Pacific (57.5\% having scores between $8-11 \%$ and $48 \%$, with scores of more than 12) and North America (35\% having scores between $8-11 \%$ and $24 \%$, with scores of more than 12; figure 4 ). In children, full data on the adapted SOFA score were available in only 122 of 220 patients aged less than 10 years, with only 2 dying, leading to an inability to perform association studies between organ dysfunction and hospital

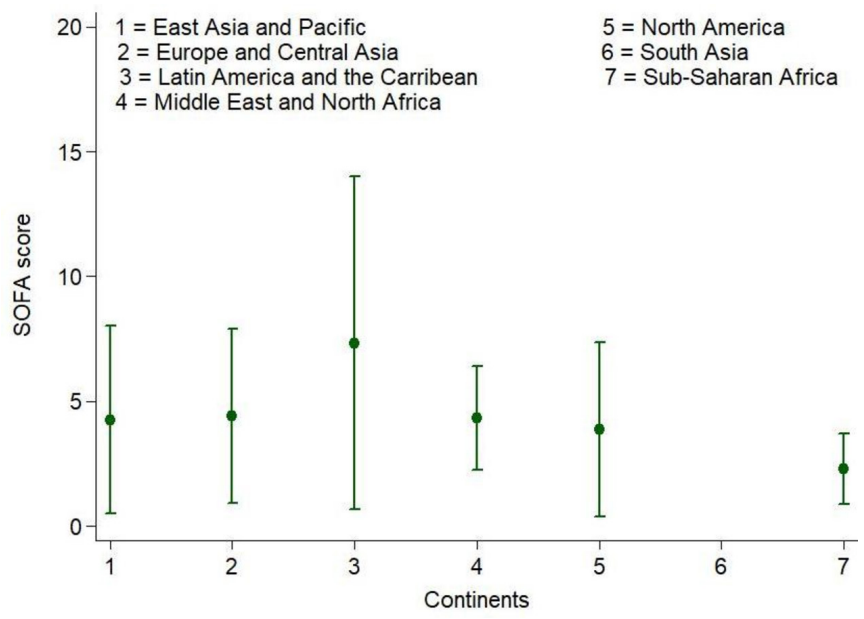

Figure 4 Sequential Organ Failure Assessment (SOFA) score by region $(n=560)$.
Table 2 Multivariable logistic regression for mortality

\begin{tabular}{|c|c|c|c|c|}
\hline \multicolumn{5}{|c|}{ Characteristics } \\
\hline SOFA score & OR & $P$ values & Lower Cl & Upper Cl \\
\hline $0-3$ & Ref & & & \\
\hline $4-7$ & 3.57 & 0.04 & 1.082 & 11.813 \\
\hline $8-11$ & 2.95 & 0.14 & 0.711 & 12.230 \\
\hline $12+$ & 12.74 & 0.001 & 2.864 & 56.694 \\
\hline Age & 1.03 & 0.001 & 1.013 & 1.049 \\
\hline \multicolumn{5}{|l|}{ Gender } \\
\hline Female & Ref & & & \\
\hline Male & 1.75 & 0.17 & 0.783 & 3.927 \\
\hline \multicolumn{5}{|l|}{ Comorbidities } \\
\hline 0 & Ref & & & \\
\hline 1 & 0.60 & 0.47 & 0.144 & 2.453 \\
\hline 2 & 1.82 & 0.29 & 0.599 & 5.509 \\
\hline 3 & 3.36 & 0.08 & 0.859 & 13.152 \\
\hline $4+$ & 3.41 & 0.08 & 0.861 & 13.486 \\
\hline \multicolumn{5}{|c|}{ Length of stay (days) } \\
\hline 0 & Ref & & & \\
\hline $1-7$ & 1.29 & 0.68 & 0.388 & 4.298 \\
\hline $8-14$ & 1.72 & 0.50 & 0.353 & 8.330 \\
\hline $15+$ & 3.53 & 0.09 & 0.842 & 14.828 \\
\hline
\end{tabular}

Ref, reference; SOFA, Sequential Organ Failure Assessment.

mortality. Missing complete data for organ dysfunction $(n=122)$ were exclusively focused on lower income regions, with greater missing data among children.

Univariate and multivariate logistic regressions were used to assess the association between mortality and main a priori proposed risk factors or adjusting for potential confounders (table 2). We identified that SOFA score at admission and increasing age were independent predictors of hospital mortality across all age groups, where SOFA scores were available. After adjusting for potential confounders, increasing SOFA score was associated with increased mortality, when compared with those with a SOFA score $\leq 3$ : SOFA $4-7$ $(\mathrm{OR}=3.57$ (95\% CI 1.08 to 11.81$), \mathrm{p}=0.04)$, SOFA $8-11$ $(\mathrm{OR}=2.95$ (95\% CI 0.71 to 12.23$), \mathrm{p}=0.14)$ and SOFA $\geq 12 \quad(\mathrm{OR}=12.74 \quad(95 \%$ CI 2.86 to 56.69$), \mathrm{p}=0.001)$. There was a $3 \%$ increased risk of mortality for every yearly increase in age (adjusted $\mathrm{OR}=1.03$ (95\% CI 1.01 to 1.05$), \mathrm{p}<0.001$ ). Analysis by year showed no significant differences between analyses restricted to 2016 or 2017 seasons.

The variability in case fatality proportions in our data set, particularly among children, in addition to patient-specific variables, is likely due to a variety of factors, including intensive care unit admission criteria,${ }^{20}$ the availability of interventions ${ }^{21}$ and the presence of reporting bias in this convenience sample, particularly as it relates to the sparse data sources for 
acute care in lower income regions and the known disease burden in those regions. ${ }^{\text {The }}$ Thepwise increase in mortality by age in our data set reflects this surprisingly low mortality in the $<5$-year-old cohort. The lack of complete severity-of-illness data from lower income regions impedes the ability to effectively understand this counterintuitive result.

\section{FROM LOCAL TO GLOBAL DATA ON SEVERE ACUTE RESPIRATORY INFECTIONS}

The global SPRINT-SARI programme has a primary purpose of testing the clinical research infrastructure and network logistics in varied settings. We demonstrated that presentation, management and outcomes for patients with SARI vary substantially according to global region. The implications of this variation are large, and suggest a need for ongoing, prospective data collection at a global scale to ensure a better understanding of patient characteristics, specific SARI risk factors and the importance of a reliable risk adjustment method for international comparative studies. Further, the use of organ dysfunction, as measured by a SOFA score collected on presentation for critically ill patients with SARI in disparate settings, appears promising. This deserves further work for risk stratification and early outbreak severity predictions, particularly in children where our data are limited.

Given previously documented variability in application of case definitions and to different care areas (eg, hospital wards, intensive care units, emergency departments), differential inclusion of patients with SARI is an ongoing risk during outbreaks, emphasising the need for standardised operating procedures for clinical data reporting. ${ }^{22}$ We have compiled standardised open-access tools at isaric.tghn.org/sprint-sari to assist in this goal. The data reported in this manuscript serve to demonstrate the tools; however, without a truly representative sample of hospitals and regions, accurate comparisons cannot be made.

\section{LIMITATIONS}

There are a number of further limitations to this work. The first is the nature of the data collected. Given its primary objective of being a research preparedness exercise, data verification audits were not exhaustively performed, and small numbers in some regions may lead to unstable proportions during comparisons. The patients that were enrolled in the study may not be representative of all critically ill patients with SARI, based on the period of data collected and location of participating hospitals. Second is the lack of a formal denominator to assess the impact of SARI on hospital-level or population-level burden of disease, or to generate estimates of disease incidence. Capturing hospital-wide activity requires substantially larger research resources at participating institutions, and there is variation as to the screening processes present at participating institutions. Third, integrating alternate patient-centred outcomes such as quality of life or functional status on discharge $\mathrm{e}^{2324}$ would provide valuable data; however, this data collection is beyond the scope of this work and will be further integrated as programmes continue to scale. Given the mostly unfunded nature of this work at the site level, these expansions can only occur with established operational support. Finally, the availability and definitions of intensive care units are variable across regions, and this impacts any discussion on interventions received, such as dialysis or mechanical ventilation. ${ }^{25}$

\section{CONCLUSIONS}

We have shown that it is feasible for a global network of hospitals to collect standardised clinical data variables on critically ill adults and children with SARI. While severity of illness and age are associated with mortality, we have also highlighted substantial variability in pathogens, management strategies and clinical outcomes according to global regions. This emphasises the importance of collecting a globally applicable severity of illness score at admission for adults and children in order to perform valid international comparative studies of patients with SARI and for future outbreak severity assessments. Future work in documenting SARI care and outcomes around the world requires ongoing, standardized prospective data collection in varied regions, particularly among children and other vulnerable populations.

Collaborators Vann Mich, Khmer Soviet Friend Hospital. Yaty Pho, Khmer Soviet Friend Hospital. Sotharith Bory, Calmette Hospital and University of Health Sciences. Mich Vann, Khmer-Soviet Friendship Hospital and University of Health Sciences. Bunlor Teav, Takeo Provincial Hospital. Leakhann Som, National Pediatric Hospital. Mikko J Jarrvisalo, Turku university hospital, ICU. Anni Pulkkinen, Central Hospital of Central Finland. Anne Kuitunen, Tampere University Hospital. Tero Ala-kokko, Oulu University Hospital, Research Group of Anesthesiology, Surgery and Intensive Care Medicine. Sari. Melto, South Karelia Central Hospital. Thomas DAIX, Reanimation polyvalente, CHU Dupuytren, Limoges, France and Inserm CIC 1435, CHU Dupuytren, Limoges, France. Francois Philippart, Intensive Care Unit. Marchalot Antoine, Dieppe General Hospital. Kelly Tiercelet, Groupe hospitalier Paris Saint Joseph. Cedric Bruel, Groupe hospitalier Paris Saint Joseph. BRUYERE Remi, Centre Hospitalier Fleyriat. Sedillot Nicholas, Centre Hospitalier Fleyriat. Shidasp SIAMI, General Intensive Care Medicine, Sud Essonne Hospital Etampes. Marchalot Antoine, Centre Hospitalier Dieppe. Taimon Fabienne, Service de Medecine Intensive et Reanimation, Rouen University Hospital (G.B.), and Normandie University, Universite de Rouen, U1096, Rouen University Hospital. Philippart, Groupe hospitalier Paris Saint joseph. Raomi Bruyere, Service de reanimation. Centre Hospitalier Fleyriat. Xavier Forceville, Grand Hopital de I'Est Francilien. Simon Erickson, Perth Children's Hospital. Lewis Campbell, Royal Darwin Hospital. Ravikiran Sonawane, Rockingham General Hospital. John Santamaria, St Vincent's Hospital (Melbourne). Mark Kol, Concord Hospital. Shally Awasthi, King George's Medical University. Jeff Powis, Michael Garron Hospital. Richard Hall, Dalhousie University. Anne E McCarthy, University of Ottawa and the Ottawa Hospital. Philippe Jouvet, Ste-Justine Hospital and Research Center. Mary Anne Opavsky, Joseph Brant Hospital. Elaine Gilfoyle, University of Calgary. Nataly Farshait, Humber River Hospital. Dori-Ann Martin, University of Calgary. Donald Griesdale, Department of Anesthesiology, Pharmacology \& Therapeutics Department of Medicine, Divisions of Critical Care Medicine \& Neurology University of British Columbia. Kevin Katz, North York General Hospital. Aaron J. Ruberto, Queen's University \& Kingston Health Sciences Centre. Francois Martin Carrier, Centre Hospitalier de I'Universite de Montreal. Francois Lamontagne, Universite de Sherbrooke. John Muscedere, Queens University. Asgar Rishu, Sunnybrook Health Sciences Centre. Wai Ching Sin, 
Department of Adult Intensive Care Unit, Queen Mary Hospital. Wallace Chun Wai Ngai, Department of Adult Intensive Care Unit, Queen Mary Hospital. Paul Young, Medical Research Institute of New Zealand. Dr Annette Forrest, Waikato Hospital. Alex Kazemi, Middlemore Hospital. Seton Henderson, Christchurch Hospital. Troy Browne, Tauranga Hospital. Anusha Ganeshalingham, Starship Hospital. Rachael McConnochie, Department of Critical Care Medicine, Auckland City Hospital. Jae Hwa Cho, Yonsei University. Tai Sun Park, Hanyang University Guri Hospital. Yun Su Sim, Hallym University Kangnam Sacred Hospital. Youjin Chang, Inje University, College of Medicine, Sanggye Paik Hospital. Heung Bum Lee, Chonbuk National University Hospital. Seung Yong Park, Chonbuk National University Hospital. Wai Ming Chan, Department of Adult Intensive Care Unit, Queen Mary Hospital, Hong Kong. Won-Yeon Lee, Yonsei University Wonju College of Medicine. David J. Wallace, University of Pittsburgh School of Medicine. Derek C. Angus, University of Pittsburgh School of Medicine. Anthony G Charles, University of North Carolina at Chapel Hill. H Rogier van Doorn, Oxford University Clinical Research Unit. Nguyen Van Kinh, National Hospital for Tropical Diseases. Nguyen Vu Trung, National Hospital for Tropical Diseases. Meghan Prin, Columbia University College of Physicians \& Surgeons. Theogene Twagirumugabe, University of Rwanda /College of Medicine and Health Sciences. Olivier Felix Umuhire, Department of Anesthesia, Emergency Medicine and Critical Care. University of Rwanda. Habarurema Sylvain, Centre hospitalier Universitaire de Butare(CHUB). Eman Al Qasim, King Saud bin Abdulaziz University for Health Sciences, King Abdullah International Medical Research Center. Jean-Michel Heraud, Institut Pasteur de Madagascar. Mihaja Raberahona, University Hospital Joseph Raseta Befelatanana. Joelinotahiana Hasina Rabarison, Insttut Pasteur de Madagascar. Santiago Perez Patrigeon, Instituto Nacional de Ciencias Medicas y Nutriciễ ${ }^{3}$ Salvador SubirÃjn. Alejandra Ramirez-Venegas, Instituto Nacional de Enfermedades Respiratorias Ismael Cosio Villegas. Javier Araujo Meléndez, Hospital Central "Dr. Ignacio Morones Prieto". M. Lourdes Guerrero, Instituto Nacional de Ciencias Medicas y Nutricion Salvador Zubiren. Ivan Mambule, Clinical Infection, Microbiology and Immunology, Institute of Infection and Global Health University of Liverpool and Malawi-Liverpool-Wellcome Trust, Clinical Research Programme. Otieno George Ochieng, Kijabe Mission Hospital. Behzad Nadjm, Imperial College Healthcare NHS Trust, GBR. Iris Wai Sum Li, Queen Mary Hospital; School of Public Health, the University of Hong Kong. Won-II Choi, Department of Medicine, Keimyung University, Dongsan Hospital. Komurian-Pradel Florence, Fondation Merieux. Yaseen M Arabi, King Saud bin Abdulaziz University for Health Sciences, King Abdullah International Medical Research Center, Intensive Care Department, King Abdulaziz Medical City. T. Eoin West, University of Washington. Elisabeth D Riviello, Harvard Medical School and Beth Israel Deaconess Medical Center. Rachael Parke, Cardiothoracic and Vascular ICU, Auckland City Hospital. AnnaneE Djillali, Raymond Poincare Hospital (APHP) Unievrsity of Versailles SQY/University Paris Saclay. Robert Fowler,

Interdepartmental Division of Critical Care Medicine, University of Toronto. Srinivas Murthy, Department of Pediatrics. Alistair Nichol, University College Dublin / Monash University. Allen C Cheng, School of Public Health and Preventive Medicine, Monash University. Calum Semple, University of Liverpool. Maya George, Australian and New Zealand Intensive Care Research Centre, Monash University. Miia Valkonen, University of Helsinki and Helsinki University Hospital. Colin McArthur, Auckland City Hospital (DCCM 82). Gail Carson, University of Oxford. Genevieve 0 'Neill, Australian and New Zealand Intensive Care Research Centre, Monash University. J. Perren Cobb, University of Southern California. Jake Dunning, University of Oxford; Imperial College London. Jean-Daniel Chiche, Hopitaux Universitaire Paris Centre, site Cochin. Jin-Won Huh, ASAN Medical Center. John Marshall, St. Michael's Hospital. Jordi Rello, Ciberes \& Vall d'Hebron University Hospital, Barcelona, Spain. Julia Guillebaud, Institut Pasteur de Madagascar. Norosoa Razanazatovo, Institut Pasteur de Madagascar. Juilett Wambura Otieno, KEMRI-Wellcome Trust Research Programme. Karen Green, Toronto Invasive Bacterial Diseases Network. Kathy Rowan, Intensive Care National Audit and Research Centre. John Kenneth Baillie, Roslin Institute, University of Edinburgh. Laura Merson, Infectious Diseases Data Observatory, Oxford, UK; Centre for Tropical Medicine \& Global Health, Nuffield Department of Medicine, Oxford University, Oxford UK. Li Yang Hsu, National University of Singapore. Michael D. Christian, Essex \& Herts Air Ambulance Trust. Miia Valkonen, Helsinki University Central Hospital. Moritoki Egi, Kobe University Hospital. Nahoko Shindo, World Health Organization. Peter Horby, University of Oxford. Raul Pardinaz-Solis, Nuffield Department of Medicine, University of Oxford. Sebastián Ugarte Ubiergo, Universidad Andrés Bello. Steve AR Webb, Monash University. Timothy M. Uyeki, Influenza Division, Centers for Disease Control and Prevention, Atlanta, Georgia USA. Anthony C Gordon, Imperial College London. David L Paterson, University of Queensland Centre. Dean Everett, University of Edinburgh, The Queens Medical Research Institute and Malawi-Liverpool-Wellcome Trust, Clinical Research Programme. Evangelos J. Giamarellos-Bourboulis, National and Kapodistrian
University of Athens, Medical School. Kajsa-Stina Longuere, University of Oxford. David Maslove, Queens University. Eric Ohuma, Oxford University. Gloria Crowl, Michael Garron Hospital. Theresa PedutemHumber, River Hospital. Edward EllazarHumber, River Hospital. Ilona Bahinskaya, University Health Network TGH MOT. Joan Osbourne-Townsend, Humber River Hospital. Andrew Bentley, University of Manchester. Ingeborg Welters, University of Liverpool. Nadia Malik, MountSinai Hospital/ William Osler Health Centre. Dr T S Browne, Tauranga Hospital. Jennifer Goodson, Tauranga Hospital. Vinaya Mahesh, North York General Hospital.

Contributors All authors, listed in the attached sheet, made substantial contributions to the design and data analysis of this work, provided comments in drafting this manuscript, approved the final version submitted, and agree to be accountable for all aspects of the work.

Funding We acknowledge support from the National Health and Medical Research Council in Australia, the Australia New Zealand Intensive Care Society Clinical Trials Group and the Seventh Framework Program in Europe, which have facilitated the progress that has been made for central project infrastructure. Data collection was funded locally by local research coordinators and investigators, including the International Respiratory and Severe IIIness Center, University of Washington. The funding sources have no role in the study design, data collection or report writing

Disclaimer The findings and conclusions in this report are those of the authors and do not necessarily represent the official position of the U.S. Centers for Disease Control and Prevention.

\section{Competing interests None declared.}

Patient consent Required at specific sites.

Ethics approval The study has been approved by all relevant ethics required at specific sites. Institutional approvals varied according to the hospital and nation, with countries such as the UK collecting data as part of pre-existing preparedness exercises and test activations of rapid response protocols.

Provenance and peer review Not commissioned; externally peer reviewed.

Data sharing statement All data collected have been de-identified and will be made available to collaborators upon request after approval by the SPRINT-SARI management committee. All research tools, including case report forms, protocols and data dictionaries, are available at sprintsari.org for others to adapt and use.

Open access This is an open access article distributed in accordance with the Creative Commons Attribution Non Commercial (CC BY-NC 4.0) license, which permits others to distribute, remix, adapt, build upon this work non-commercially, and license their derivative works on different terms, provided the original work is properly cited, appropriate credit is given, any changes made indicated, and the use is non-commercial. See: http://creativecommons.org/licenses/by-nc/4.0

\section{REFERENCES}

1. GBD 2015 LRI Collaborators. Estimates of the global, regional, and national morbidity, mortality, and aetiologies of lower respiratory tract infections in 195 countries: a systematic analysis for the Global Burden of Disease Study 2015. Lancet Infect Dis 2017;17:1133-61.

2. Sakr Y, Ferrer R, Reinhart K, et al. The intensive care global study on severe acute respiratory infection (IC-GLOSSARI): a multicenter, multinational, 14-day inception cohort study. Intensive Care Med 2016;42:817-28.

3. Baden LR, Rubin EJ, Morrissey S, et al. We can do better improving outcomes in the midst of an emergency. $N$ Engl $\mathrm{J}$ Med 2017;377:1482-4.

4. Rojek AM, Horby PW. Modernising epidemic science: enabling patient-centred research during epidemics. BMC Med 2016;14:212.

5. Lurie N, Manolio T, Patterson AP, et al. Research as a part of public health emergency response. N Engl J Med 2013;368:1251-5.

6. Fowler R, Rishu A, Marinoff N, et al. Global research preparedness for outbreak-related critical illness. J Crit Care 2017;440:272.

7. World Health Organization. Weekly epidemiological record. Geneva: World Health Organization, 2017: 761-80.

8. World Health Organization. Weekly epidemiological record. Geneva: World Health Organization, 2016: 621-4.

9. Gobat NH, Gal M, Francis NA, et al. Key stakeholder perceptions about consent to participate in acute illness research: a rapid, systematic review to inform epi/pandemic research preparedness. Trials 2015;16:591.

10. Vincent JL, Moreno R, Takala J, et al. The SOFA (Sepsis-related Organ Failure Assessment) score to describe organ dysfunction/ failure. On behalf of the working group on sepsis-related problems of 
the European society of intensive care medicine. Intensive Care Med 1996;22:707-10.

11. Matics TJ, Sanchez-Pinto LN. Adaptation and validation of a pediatric sequential organ failure assessment score and evaluation of the sepsis-3 definitions in critically ill children. JAMA Pediatr 2017;171:e172352.

12. The World Bank Group, 2018. World bank country and lending groups. Available from: https://datahelpdesk.worldbank.org/ knowledgebase/articles/906519-world-bank-country-and-lendinggroups [Accessed 22 Feb 2018]

13. Baker AW, Edmond MB, Herwaldt LA, et al. Real-time surveillance of influenza morbidity: tracking intensive care unit resource utilization. Ann Am Thorac Soc 2017;14:1810-7.

14. Bertolini $G$, Nattino $G$, Langer $M$, et al. The role of the intensive care unit in real-time surveillance of emerging pandemics: the Italian GiViTI experience. Epidemiol Infect 2016;144:408-12.

15. Pai NP, Vadnais C, Denkinger C, et al. Point-of-care testing for infectious diseases: diversity, complexity, and barriers in low- and middle-income countries. PLoS Med 2012;9:e1001306.

16. GLOPID-R Secretariat. Global research collaboration for infectious preparedness GloPID-R, 2016.

17. World Health Organization, 2018. World health organization model list of essential in vitro diagnositics. Available from: http://www.who. int/medical_devices/diagnostics/EDL_ExecutiveSummary_15may. pdf [Accessed 11 Jun 2018].

18. Breiman RF, Cosmas L, Njenga M, et al. Severe acute respiratory infection in children in a densely populated urban slum in Kenya, 2007-2011. BMC Infect Dis 2015;15:95.
19. Cohen C, Walaza S, Moyes J, et al. Epidemiology of severe acute respiratory illness (SARI) among adults and children aged $\geq 5$ years in a high HIV-prevalence setting, 2009-2012. PLoS One 2015;10:e0117716.

20. Wunsch $\mathrm{H}$, Angus DC, Harrison DA, et al. Variation in critical care services across North America and Western Europe. Crit Care Med 2008;36:2787-e8.

21. Baelani I, Jochberger S, Laimer T, et al. Availability of critical care resources to treat patients with severe sepsis or septic shock in Africa: a self-reported, continent-wide survey of anaesthesia providers. Crit Care 2011;15:R10.

22. Duggal A, Pinto R, Rubenfeld G, et al. Global variability in reported mortality for critical illness during the 2009-10 influenza a(h1n1) pandemic: a systematic review and meta-regression to guide reporting of outcomes during disease outbreaks. PLOS One 2016;11:e0155044

23. Honselmann KC, Buthut F, Heuwer B, et al. Long-term mortality and quality of life in intensive care patients treated for pneumonia and/ or sepsis: Predictors of mortality and quality of life in patients with sepsis/pneumonia. J Crit Care 2015;30:721-6.

24. Pollack MM, Holubkov R, Funai T, et al. Pediatric intensive care outcomes: development of new morbidities during pediatric critical care. Pediatr Crit Care Med 2014;15:821-7.

25. Marshall JC, Bosco L, Adhikari NK, et al. What is an intensive care unit? A report of the task force of the World Federation of Societies of Intensive and Critical Care Medicine. J Crit Care 2017;37:270-6. 